\title{
ORBITALS IN GENERAL CHEMISTRY, PART III: CONSEQUENCES FOR TEACHING
}

\author{
Guy Lamoureux ${ }^{\mathrm{a}, \mathrm{b}, *, @}$ and John F. Ogilvie ${ }^{\mathrm{a}, \mathrm{c}, \mathrm{d}}$ \\ aUniversidad de Costa Rica, Escuela de Química, 11501-2060 San Pedro de Montes de Oca, San José, Costa Rica \\ ${ }^{b}$ Centro de Investigaciones en Productos Naturales, 11501-2060 San Pedro de Montes de Oca, San José, Costa Rica \\ ${ }^{c}$ Centre for Experimental and Constructive Mathematics, Department of Mathematics, Simon Fraser University, 8888 University \\ Drive, Burnaby, British Columbia V5A 1S6 Canada \\ 'Institute of Quantum Physics, Irkutsk National Research Technical University, 83 Lermontov Street, Irkutsk 664074, Russian \\ Federation
}

Recebido em 14/06/2020; aceito em 22/09/2020; publicado na web em 28/10/2020

\begin{abstract}
In Part III of this series, we undertake a critique of the nature of application of orbitals to describe or to explain the structure and the binding within molecules and materials. Teaching orbitals in introductory chemistry presents five dilemmas that cannot be easily resolved. We thus conclude, based on mathematical realties, that orbitals are not essential until advanced courses. Even in advanced courses, we question the traditional choice of presenting an inadequate set of orbitals. When one recognizes, in a context of general chemistry, the irrelevance of orbitals as algebraic formulae to the observable properties and reactions of chemical substances, one can readily proceed to teach appropriate content effectively without invoking orbitals or analogous entities, based on our actual experience in teaching general chemistry over the years.
\end{abstract}

Keywords: teaching general chemistry; orbitals; quantum mechanics; molecular structure.

\section{INTRODUCTION}

There is no place for dogma in science. The scientist is free, and must be free to ask any question, to doubt any assertion, to seek for any evidence, to correct any errors. J. R. Oppenheimer

There are natural tendencies for an experimental chemist to believe that orbitals are justified theoretically and for a theoretician to believe that orbitals are proved by experiment. A philosophical chemist might debate the existence of orbitals, and whether one should believe in orbitals. ${ }^{1}$ To continue to teach orbitals in introductory chemistry, a pragmatic chemist should, however, be convinced that orbitals are pedagogically effective. A student who is unable to understand or to apply orbitals to solve problems resorts to memorization, which is the current state of teaching and learning introductory chemistry. ${ }^{2}$

Our arguments below pertain to a general-chemistry class that contains students of diverse intended-major subjects for whom basic chemistry is required - those institutions that separate future specialist chemistry students into a separate course or section likely determine their own curricula. Based on the information presented in Parts I and II, we argue that, if orbitals are eliminated at the basic level, the students might become uninformed; if orbitals continue to be taught as they are, the students definitely become misinformed. This orbital-free approach is not about 'dumbing-down' the course - the time and energy formerly applied to teach orbitals in a pseudoquantum-mechanical context can serve to introduce recent chemical developments, or to teach significant chemical concepts that would otherwise become neglected. We focus in this article on alternative ways to prevent the propagation of misinformation.

The issue here is the use of orbitals in qualitative explanations of atomic and molecular structure and properties. Common textbooks of general chemistry customarily devote much space and importance to orbitals as if they were somehow more, or other, than mere algebraic

*e-mail: guy.lamoureux@ucr.ac.cr formulae or mathematical functions applicable to only the hydrogen atom, which is a logical fallacy. Although in Parts I and II we touted the reasonable definition of orbital in the textbook by Brown et al., ${ }^{3}$ most textbooks descend into the morass of unwarranted explanations involving orbitals. The incontestable principle according to which one must invariably abide is that electrons are fundamentally indistinguishable. ${ }^{4}$ The pertinent experimentally observable quantity is the total density of electronic charge in the vicinity of one or other atomic nucleus in a molecule or within a unit cell of a crystal. Any attempt to partition that density relies on arbitrary criteria and is thereby subject to error, invalid conclusions and refutable inferences.

As discussed earlier, we are not completely anti-orbital; delay does not mean denial. Orbitals are a useful, but flawed, model for advanced chemistry. Orbitals will continue to serve, in research and in quantum chemistry, in the foreseeable future for a prediction and explanation of phenomena in chemistry and material science. Physical chemistry (subsequently in the undergraduate or graduate program) might continue to provide students with the awe of the Schroedinger equation - and its limitations. We repeat explicitly no objection to the use of orbitals in basis sets for, or other uses in, actual calculations, even though they might be superfluous; for instance, calculations based on the amplitude function of James and Coolidge contained no orbital. ${ }^{5}$ Calculations according to density-functional theory can be made accurately with software for electronic structure free of orbitals - no orbital is involved at any stage of the calculations, but the results for observable properties have accuracy comparable with that of alternative software for quantum chemistry. ${ }^{6}$ Quantum-chemical calculations are, however, irrelevant, and far remote from the teaching and practice of general chemistry; our concern is the suitability of invoking orbitals in teaching general chemistry to undergraduates in their first year of study after secondary school. Our content and arguments have, understandably, implications that are necessarily applicable to preceding and following instruction in chemistry.

We hence contend that there is a great disparity between the use of quantum-chemical software for research and the presentation of quantum theory, in a superficial guise of orbitals, to provide a 
rationalization of topics in first-year chemistry. We have no quarrel with the use of software to provide visualizations of molecules and their interactions; this visualization can be productively implemented without orbitals in an introductory course, without a complete (and superfluous) understanding of how the software works. According to our experience, chemistry has become more difficult to teach and less understood by the public, in large part due to the complications of orbitals; other authors have confirmed this impression. ${ }^{\text {? }}$

\section{DISCUSSION}

\section{Are orbitals essential models in introductory chemistry?}

A common justification for the teaching of orbitals is that this model is useful and simple to illustrate some basic themes about chemistry: "The concept of orbital is a key concept in teaching: it is used to explain bonding, chemical structure, and reactivity. Therefore, chemistry educators cannot do without such a concept in their teaching." 8 Are orbitals really key models, useful, simple, essential at the introductory level?

Gillespie disagreed with the essential and simple aspects of orbitals: "Concepts such as hybrid and molecular orbitals and the equations of thermodynamics are too abstract and too difficult for an introductory course. Moreover, they are unnecessary." Sánchez-Gómez et al. regarded orbitals, instead of being useful, as an impediment: "We think that 'atomic orbitals' are more a problem than a help in teaching chemistry. Perhaps getting rid of all these lexical false friends is a good starting point for designing an integrated chemistry curriculum." 10 Tsaparlis stated the same opinion more explicitly: "We must seriously consider alternative ways that avoid the orbitals at the high school and general-chemistry level." 11

The function of a model in science is to provide a rationale that unifies a series of apparently unrelated facts, but such a model must not be contrary to fundamental chemical and physical laws. The application of orbitals to describe qualitatively the properties of atoms with more than one electron is a quintessential instance of such a flouting of physical laws, because these electrons are supposed to be attracted through a Coulombic force to the atomic nucleus but not to be subject to a Coulombic repulsion from one another. The utility of the orbital model is weakened because there is no experimental evidence for the form of any explicit orbital, ${ }^{4}$ and, as we have shown in Part II, the theoretical basis of the use of orbitals to explain structures is suspect. ${ }^{7}$

In Part I we demonstrate that the conventionally taught orbitals are neither indispensable nor fundamental; every topic that might involve these artefacts of wave mechanics can be presented alternatively and discussed, at an introductory university level, without orbitals. The mathematical realities presented in Part II create a logical basis to consider orbitals unnecessary. The focus on only one 'shape' of orbitals (implied in the idea that there is only one Schroedinger equation for the hydrogen atom) causes misconceptions. Unless one is willing and able to treat orbitals in all these sets in general chemistry, it is unwise to waste time teaching the results of any one set, only to teach subsequently that these results are not unique. We contend that orbitals are neither necessary (intrinsic) nor essential (extrinsic) in introductory chemistry.

\section{Five dilemmas in teaching orbitals}

Our investigation into the teaching of orbitals has led to the emergence of five dilemmas that are important to resolve. Some of these have been indicated earlier, ${ }^{9}$ but the complications have expanded in the succeeding decades. Dilemmas \#1 - \#3 relate to the practical value (time versus learning) of orbitals to beginning students. Dilemmas \#4 and \#5 relate to the limitations of the average first-year student.

Dilemma \#1 - More students are enrolled in general chemistry in the twenty-first century than ever before, but the fraction of students with chemistry as major subject among total university students has decreased. ${ }^{12}$ Whereas it is a noble objective to treat all students as future chemists, the reality is that general chemistry should educate all to a particular level of understanding and appreciating science. ${ }^{13}$ The problem is to teach a course that treats all students alike while recognizing their differences. It is reported that non-chemistry majors will never use orbitals, or quantum mechanics, in their future studies. ${ }^{14}$ What about the students who are not decided on their major subject? As Gillespie wrote:

"I strongly suspect that the students who do well in elementary chemistry courses are those who are prepared to learn all this material [i.e. about orbitals] by heart and to regurgitate it in the examination or class test. The really bright students who make a serious attempt to understand the material will either waste so much time, in what is for them an impossible task, that they will fail the examination or, more probably, they will realize that it is not possible for them to understand it and to do the minimum needed to pass the examination, and then move into biology, physics or some other subject."15

From another perspective, orbital-based explanations create difficulties for many students with ultimate major subjects other than chemistry in an introductory course that is considered required for graduation. "Both chemistry teachers and non-major students appear to agree that freshman chemistry may well be the most problematic traditional science discipline taught in the first year of college - as far as students' misunderstandings, learning difficulties and misconceptions are concerned...It appears to be agreed upon that the roots of many difficulties and misconceptions that freshman students have lie in deficient understanding of the complicated, abstract and non-intuitive quantum model of the atom." 16

Dilemma \#2 - The size and density of the chemistry textbook has abutted against the time constraints to teach the material. A tendency to include the latest representations based on quantum chemistry, while retaining, or to the detriment of, all previous information, in chemistry textbooks has caused a major surge in the size and cost of these books. In 1900 a chemistry textbook for a year course comprised about 200 pages $;{ }^{15}$ a typical current textbook extends to more than 1200 pages, including multi-color images of orbitals. Can an instructor legitimately expect a student to read the entire textbook?

With a trend of reducing general chemistry to a one-semester course for all, ${ }^{17}$ some topics must be eliminated. It is suggested that the quality, not the quantity, of content should be employed to decide the limitations. In discussing a reform of the chemistry textbook, Sienko and Plane defined rules to guide a decision about what to include. ${ }^{12}$ The two most important rules follow.

- Write nothing that is not completely correct.

- Do not oversimplify, instead omit. 
These rules would thoroughly eliminate orbitals! Future students with diverse backgrounds and career choices require essential chemical information without oversimplified and confusing models. "We need to focus on meaningful learning, discourage rote learning, aim at coherent understanding and anticipate pre-conceptions. These can be achieved only by integrated, in-depth coverage of the topics." 18

Dilemma \#3 - In general chemistry, quantum-mechanical concepts are typically presented as pictorial representations, generally because of the limitations of textbook, curriculum and instructor. "For many chemists, and undoubtedly most students, almost all reasoning with orbitals is spatial and involves diagrammatic manipulations rather than algebraic ones."19 This visual learning of sub-microscopic models is fraught with pedagogical danger. ${ }^{20}$ Not only do students hence acquire a false visual understanding of orbitals in general chemistry but also this misinformation affects chemistry students in their subsequent studies. "Students arrive at the quantum chemistry course carrying with them from previous instruction a number of misconceptions and incomplete knowledge about quantum-chemical concepts. This is attributed mainly to the elementary, imprecise and mostly pictorial coverage of the relevant concepts." ${ }^{21}$

Dilemma \#4 - One can teach quantum mechanics with a mathematical level, exemplified in Part II, above most, if not all, college students, or one can accept the mathematical limitations and minimize understanding of the topic. The best presentation of quantum mechanics, including quantum numbers, orbitals and electron configuration, is based on a mathematical model of the atom. ${ }^{22}$ From a student's perspective, quantum-mechanical concepts are difficult to grasp in a non-mathematical fashion, ${ }^{11}$ which is only reasonable because quantum mechanics is fundamentally a collection of mathematical methods, or algorithms, applicable to calculations of systems on an atomic scale. ${ }^{23}$ For this reason orbitals that are fundamentally algebraic formulae are disingenuously discussed in general chemistry as divorced completely from their mathematical origins.

When reading this article, please consider the mathematical abilities of an average introductory student as further evidence that orbitals are inappropriate within instruction at this level. A recent survey of basic arithmetic (non-calculus) skills of a first-year general-chemistry group provided an average grade $52 \%$, with a strong correlation between arithmetic test scores and the final grade in the chemistry course. ${ }^{24}$

Dilemma \#5 - A complete presentation of orbitals must, by definition, be multiplistic ${ }^{25}$ yet most, if not all, first-year students are dualistic. ${ }^{26} \mathrm{~A}$ dualistic student seeks to minimize confusion by selecting the most 'clear' route to responding to the expected question and rejects 'unclear' routes learning is black or white. We show in Part II that there exist four possible, but equivalent, solutions of Schroedinger's equation for the hydrogen atom to generate atomic orbitals. Some textbooks confuse the issue further with 'orbits' from Bohr's model. ${ }^{27}$ Textbooks might then present delocalized molecular orbitals (MO), localized molecular orbitals (LMO), hybridized atomic orbitals (HAO) and valence-bond orbitals (VBO) as equivalent yet distinct representations of orbitals in molecules. A dualistic student thinks which solution is 'correct' and might attract the most points on the examination? while ignoring the other options.
An interesting survey of instructors of general chemistry in 2005 found a hesitancy to the teaching of quantum concepts. ${ }^{28}$ It summarizes the findings as due to two impediments: that the chemical instructors themselves confessed to a limited command of quantum theory (Dilemma \#3) and their belief that quantum concepts are too difficult for their students to grasp in general chemistry (Dilemmas \#4 and \#5). ${ }^{28}$

These dilemmas cannot be easily resolved. One approach to seek to increase the learning by students about orbitals in a shorter time (Dilemmas \#1 and \#2) has involved the use of technology. The current technology has naturally altered over the years, from using computers to 'view' orbitals, ${ }^{29}$ various computer software for visualization, ${ }^{30}$ internet web-pages, ${ }^{31} 3 \mathrm{D}$ printing of orbitals ${ }^{32}$ and even virtual reality. ${ }^{33}$ Most such presentations are less than ideal $;{ }^{34}$ all are incomplete ( $c f$. Part II). Moreover, one study found that visualizations on a computer in three dimensions did not help students to understand the concepts: "Summarizing, our results indicated that the students confused the characteristics of planetary and QAM [Quantum Atom Model] models even after having interacted with the visualizations... It seems that $3 \mathrm{D}$ visualizations concerning the QAM do not improve students' understanding." ${ }^{35}$ We propose that the use of technology does not address Dilemmas \#3, \#4 and \#5 - dualistic students use these tools to memorize more pictures of orbitals, perhaps with a better resolution and more three-dimensional than a textbook can present, but without achieving understanding.

These five dilemmas appear to have caused a losing situation for both instructors and students ( $c f$. Part I). It is time to accept these limitations and to expect that the gulf between a presentation of orbitals and their understanding will only increase. Considering that orbitals have been imposed on first-year chemistry students for more than 60 years and that there are still problems in their pedagogy, should one not rethink the strategy?

\section{Which set of atomic orbitals should one use for teaching purposes?}

If, despite the preceding evidence, a reader remain unconvinced that teaching with orbitals is inappropriate, that reader should ask which orbitals the teaching should include. The properties involved in their derivation can enable a rational response to that question. The orbitals according to amplitude functions in four distinct sets have clearly disparate shapes of their surfaces of constant amplitude $\psi$, as Figures 2, 4, 6 and 8 in Part II clearly demonstrate; typical qualitative applications of orbitals in general chemistry rely on those shapes, even though the overall sizes do not vary markedly for functions corresponding to a same value of energy quantum number $n$. The amplitude functions in both spherical polar and spheroconical coordinates are derived under conditions of strictly spherical symmetry - no other atom in the system and no electric field; this condition logically precludes their use in discussing a bond between a hydrogen atom and another atom, contrary to practically universal practice in the former case. The amplitude functions in paraboloidal coordinates are suitable for use in the presence of a homogeneous electric field parallel to the symmetry axis, for instance, but that condition is far from applicable to the presence of another atom along that axis.

In contrast, for amplitude functions in ellipsoidal coordinates, another atomic nucleus is perfectly acceptable at the otherwise vacant second focus of the ellipsoid; the atomic orbital of the former hydrogen atom then becomes a molecular orbital. This fact has been known since at least year $1930,{ }^{36}$ when Teller announced that property based on his calculations of the dihydrogen molecular cation, $\mathrm{H}_{2}^{+}$, but chemists have ignored that wisdom. Any use of orbitals in spherical 
polar coordinates in relation to chemical bonds, as is common practice for instance, must hence be logically unacceptable.

\section{Teaching structure without orbitals}

Dirac stated that "science is concerned only with observable things". ${ }^{37}$ The atomic centers in molecules are observable as local maxima of electronic density. Orbitals are not observable. A molecule comprises a collection of atomic nuclei and their collective associated electrons; for this reason we refrain from referring to atoms in molecules. These locations of atomic centers are most commonly defined in experiments on single crystals with the diffraction of $\mathrm{X}$-rays, which, ironically, yield the locations of atomic centers, and hence the associated atomic nuclei therein, within a unit cell detected through the local maxima of electronic density; each local maximum is attributed to an embedded atomic nucleus. Hydrogenic atomic centers are notoriously difficult to locate with X-rays because of the small scattering power of their electronic density, but diffraction with neutrons is highly successful for this purpose. As neutron diffraction is an uncommon practice, the relative positions of hydrogen atoms are in many cases imposed, based on expectations from known experimental data. The structures of molecules and crystals are hence the result of experimental determinations and should be taught from this point of view. Instead of electron configurations, which are anyhow not unique, ${ }^{4}$ the structures at the atomic (not sub-atomic) level, properties and reactions of chemical substances - elements, compounds and their mixtures, and materials - should be the main focus of attention in general chemistry.

In chemistry, the structure of molecules and materials in authenticated instances is an experimental fact, for which a theoretical prediction or justification is entirely redundant in introductory chemistry. Any such claimed prediction actually corresponds to a circular argument, in that the qualitative orbital explanation is invariably constructed to yield the observed structure. For a genuine calculation on even a large system, such that a severe limitation is imposed on the accuracy of the results, the treated system is still an ideal prototype, free from the effects of a real environment such as a solvent or substrate, and must be of invariably questionable pertinence to the results of chemical and physical experiments on a real system on a macroscopic scale.

The incompatibility of molecular structure and quantum mechanics has been recognized for many years. ${ }^{38}$ Apart from some perhaps esoteric aspects of this dichotomy, an acute understanding can be based directly on the fact that a calculation - because quantum mechanics is a collection of methods of calculation - in which the electrons and atomic nuclei are treated equitably, i.e. applying basis sets in wave mechanics to both electrons and nuclei and integrating over all coordinates, yields no structure; this effect is proved experimentally (computationally). ${ }^{39}$ The exceptions are for diatomic molecules, for which a structure is trivial, and the simplest triatomic molecules, because nuclei are invariably distinguishable from electrons. Quantum-chemical calculations are valuable adjuncts for experimental observations, but such calculation is no component of introductory chemistry.

\section{Teaching general chemistry without orbitals}

We aim to show a pragmatic chemist that teaching and learning of general chemistry is practicable without orbitals. We envisage a future in which the content is divided into modules, each linked to another, that focus on learning chemistry in a more practical way: the importance of chemistry to industry, medicine and the environment. 'How' questions should be the focus of the modules to enable one to predict properties and reactivity, with 'what' questions providing descriptions. Hence, 'How are the elements organized in a periodic chart?', 'How are organic molecules organized with functional groups?' 'How does the molecular structure affect the physical properties of a substance?'

Some 'why' questions (e.g. Why is the structure of a water molecule angular? Why is methane tetrahedral?) can be dismissed completely (these questions are, of course, open to advanced research programs). As both Weinberg and Feynman have shown, 'why' questions in science lead to never-ending explanations, with deeper deductive and more unintelligible answers. ${ }^{40}$ An explanation of a structure in terms of orbitals leads naturally to a question of why Schroedinger's equation is pertinent. Pragmatic teaching of chemistry does not concern itself with why. Students of introductory chemistry expect a narrative explanation; scientific explanations are hence commonly found unsatisfying. "We, the teachers, are unable to offer good explanations... an explanation is necessarily deductive whereas chemical science is basically inductive. This observation is highly relevant to the teaching of chemistry." ${ }^{41}$

The tetrahedral geometric disposition of hydrogenic atomic centers around a carbon atomic center in a molecule of methane in the state of least energy is a matter of observation and scientific fact, first deduced from chemical evidence and subsequently abundantly confirmed with physical data - there is no need to justify this fact retrospectively. We suggest that educators present to students, instead of pseudo-quantum-mechanical explanations, the three-dimensional structures as known data. Eliminate the onerous flow chart that students memorize and forget. ${ }^{42}$ There are several freely accessible sources of these data, mostly obtained experimentally: Wikipedia. org, molview.org, several databases and software programs. With the structure known (not predicted), the interesting part of chemistry - the chemical properties and reactions - can be investigated.

The principal emphases of introductory or general chemistry might be epitomized to be stoichiometry and chemical equilibrium, especially ionic equilibria in aqueous solution that is the basis of not only traditional analytical chemistry but even life itself. A standard precept in the effective teaching of experimental science is that there should exist a strong correlation between the concurrent lecture and laboratory components of a particular course or subject. Any discussion of atomic orbitals in lectures hence violates this precept because orbitals, as indisputable artefacts of not just one particular method of quantum mechanics but also a selected coordinate system (as delineated in Part II), are not observable quantities subject to experimental verification. The presentation of chemical and physical equilibria and associated thermodynamic and kinetic aspects provides little or no legitimate occasion for an involvement of electron configurations or orbitals but profound opportunity for experimental practice without major equipment and cost, other than for the chemicals consumed.

After conventional preliminary topics up to and including the stoichiometric trends of simple compounds based on the periodic chart of the chemical elements, a discussion of structure at a microscopic level begins, naturally enough, with the structures of simple crystals, beginning with ionic crystals and proceeding to molecular crystals. The discussion of structure proceeds to other simple binary compounds, including those containing carbon as a basis of organic chemistry.

A discussion of reactivity free of orbitals might involve a use of maps of molecular electrostatic potential. ${ }^{43}$ We avoid a comparison of molecular-orbital (MO) theory versus valence-bond (VB) theory, also a comparison of canonical MO versus localized MO (LMO) versus hybridized atomic orbitals (HAO), as the conclusions remain the same: in introductory chemistry, all presentations of orbitals 
are irrelevant and can be eliminated without affecting the quality of the education. Their omission might even improve the quality of the perceived chemical information by eliminating superfluous distraction.

As a basis for the properties, reactions and applications of chemical substances and their mixtures, the structure of molecules and materials is the primary focus of chemistry. "Syntheses, reactions, and commercial processes, physical properties, phases at various temperatures and pressures, structures, habits and solubility behaviors that we can see, smell, touch and even hear - this is material that is eminently memorable. Indeed, this is the stuff the students remember all their lives and whose immediacy can capture their imaginations." ${ }^{42}$

One must, naturally, discover a balance between experiment and theory. As quantum mechanics is anathema to molecular structure, forget about quantum mechanics and its orbital artefacts; apply instead the quantum laws or laws of discreteness ${ }^{44}$ that suffice for accurate explanations of atomic and molecular spectra across many frequency ranges. For many common purposes of calculations, such as refined molecular structures and molecular electric dipolar moments, one can apply methods of molecular mechanics, with no quantum-mechanical provenance whatsoever, instead of methods of quantum chemistry; such calculations yield results of acceptable accuracy, and likely at a great economy of computing duration, ${ }^{45}$ but this calculating activity is still alien to the teaching and practice of general chemistry.

\section{CONCLUSIONS}

To confront the teaching dilemmas of the future, we have shown that the presentation of orbitals must be revised. Because of limitations of time, mathematical ability and misconceptions, undergraduates in the first-year course can pragmatically not be expected to learn and to appreciate orbitals in all their various forms. The presentation of orbitals in all present textbooks is certainly an oversimplification and should be omitted, according to the precepts of Sienko and Plane, ${ }^{12}$ to provide an appropriate education to the students.

The existence of the above dilemmas shows that orbitals are neither necessary nor sufficient for their role in teaching chemistry. Every prediction at the introductory level based on orbitals we can show to be unnecessary for general chemistry. Orbitals have supplanted their role as models in chemistry when they serve as the only possibility for the visualization, description and explanation of simple chemistry. Orbital models are certainly not simple for students who lack the mathematical basis to understand the amplitude equations. These previous arguments have been made ad nauseum by outstanding educators and chemists, as summarized in Part I - our new data in Part II provide profound mathematical support beyond reproach and lead to an inescapable conclusion that up to $20 \%$ of the content of general chemistry, treating orbital concepts, must be revised.

If instructors of chemistry genuinely understood the mathematics underlying the production and application of the algebraic functions defined as orbitals, ${ }^{3}$ such as we have summarized, they would not teach such irrelevant material; although we concur with Gillespie ${ }^{15}$ on this matter, our conclusion is based on undeniable mathematical realities and profound understanding, not merely intuitive feeling. It is timely to jettison the ponderous yoke of orbitals and to redirect the attention of students of introductory chemistry to the reality of the structure of molecules and materials as a product of scientific experiment based on chemical and physical principles.

\section{ACKNOWLEDGEMENTS}

El Centro de Investigaciones en Productos Naturales (CIPRONA) and la Escuela de Química, Universidad de Costa Rica (UCR) provided support. We thank several students and professors at UCR for helpful discussion.

\section{REFERENCES}

1. Jenkins, Z.; Phil. Sci. 2003, 70, 1052.

2. Jensen, W. B.; J. Chem. Educ. 2014, 91, 1106.

3. Brown, T. L.; LeMay, H. E.; Brusten, B. E.; Murphy, C. J.; Woodward, P. M.; Chemistry: the Central Science, $12^{\text {th }}$ ed., Pearson: Boston, USA, 2012, pp. 220-221.

4. Truhlar, D. G.; Hiberty, P. C.; Shaik, S.; Gordon, M. S.; Danovich, D.; Angew. Chem., Int. Ed. 2019, 58, 12332.

5. Aslangul, C.; Constanciel, R.; Daudel, R.; Kottis, P.; Adv. Quant. Chem. 1972, 6, 93.

6. Chen, M.; Xia, J.; Huang, C.; Dieterich, J. M.; Hung, L.; Shin, I.; Carter, E. A.; Comp. Phys. Comm. 2015, 190, 228; Mi, W.; Shao, X.; Su, C.; Zhou, Y.; Zhang, S.; Li, Q.; Wang, H.; Zhang, L.; Wang, Y.; Ma, Y.; Comp. Phys. Comm. 2016, 200, 87.

7. Lamoureux, G.; Ogilvie, J. F.; Química Nova (2020), Orbitals in General Chemistry, Part I, in press. Lamoureux, G.; Ogilvie, J. F.; Química Nova (2020), Orbitals in General Chemistry, Part II, in press.

8. Labarca, M.; Lombardi, O.; J. Chem. Educ. 2007, 84, 187.

9. Gillespie, R. J.; J. Chem. Educ. 1991, 68, 192.

10. Sánchez-Gómez, P. J.; Martín, F.; Chem. Educ. Res. Pract. 2003, 4, 131.

11. Tsaparlis, G.; J. Chem. Educ. 1997, 74, 922.

12. Bell, J. A. In Sputnik to Smartphones: A Half-Century of Chemistry Education; Orna, M. V., ed.; ACS Publications: Washington, DC, 2015, 25.

13. Cooper, M.; J. Chem. Educ. 2010, 87, 231.

14. Hawkes, S. J.; J. Chem. Educ. 1992, 69, 178; Hawkes, S. J.; J. Chem. Educ. 2000, 77, 321.

15. Gillespie, R. J.; Chem. Can. 1976, 12, 23.

16. Zoller, U.; J. Res. Sci. Teach. 1990, 27, 1053.

17. Kennerly, W. W.; Frederick, K. A.; Sheppard, K.; J. Chem. Educ. 2020, 97, 1295.

18. Tsaparlis, G.; Res. Sci. Educ. 1997, 27, 271.

19. Woody, A. I.; Found. Chem. 2004, 6, 13.

20. Eilks, I.; Witteck, T.; Pietzner, V.; Center for Educational Policy Studies Journal 2012, 2, 125, available at https://www.pedocs.de/frontdoor. php?source_opus $=5828$, accessed in October 2020 .

21. Tsaparlis, G. In Advances in Teaching Physical Chemistry; Ellison, M., ed.; American Chemical Society: Washington, D. C., 2008, pp. 75-112.

22. Ruis, S. P.; J. Chem. Educ. 1988, 65, 720

23. Styer, D. F.; Balkin, M. S.; Becker, K. M.; Burns, M. R.; Dudley, C. E.; Forth, S. T.; Gaumer, J. S.; Kramer, M. A.; Oertel, D. A.; Park, L. H.; Rinkoski, M. T.; Smith, C. T.; Wotherspoon, T. D.; Am. J. Phys. 2002, 70, 288.

24. Williamson, V. M.; Walker, D. R.; Chuu, E.; Broadway, S.; Mamiya, B.; Powell, C. B.; Shelton, G. R.; Weber, R.; Dabney, A. R.; Mason, D.; Chem. Educ. Res. Pract. 2020, 21, 51.

25. Finster, D. C.; J. Chem. Educ. 1991, 68, 752.

26. Finster, D. C.; J. Chem. Educ. 1989, 66, 659.

27. Allred, Z. D. R.; Bretz, S. L.; J. Chem. Educ. 2019, 96, 1558.

28. http://quantumconcepts.bu.edu/dissemination.html, accessed in October 2020.

29. Allendoerfer, R. D.; J. Chem. Educ. 1990, 67, 37.

30. Kittiwutthisakdi, K.; Kueseng, K.; Walailak J. Sci. Tech. 2005 , 2, 169, available at http://wjst.wu.ac.th/index.php/wjst/article/ download/161/153, accessed in October 2020.

31. htps://winter.group.shef.ac.uk/orbitron, accessed in October 2020.

32. Griffith, K. M.; Cataldo, R. D.; Fogarty, K. H.; J. Chem. Educ. 2016, 93 , 1586.

33. Lang, A. S.; Kobilnyk, D.; J. Virtual Worlds Res. 2009, 2, 4. 
34. Barradas-Solas, F.; Gómez, P. J. S. Chem. Educ. Res. Pract. 2014, 15, 311.

35. Kontogeorgiou, A.; Bellou, J.; Mikropoulos, T. A. In Contributions from Science Education Research; Pintó, R., Digna, C., ed.; Springer: New York, 2007, pp. 465-475.

36. Teller, E.; Z. Phys. 1930, 61, 458.

37. Dirac, P. A. M.; The Principles of Quantum Mechanics. Oxford University Press: London, 1967, p. 3.

38. Sutcliffe, B. T.; Woolley, R. G. I.; arXiv Preprint 2020, DOI: arXiv:2003.06599.
39. Cafiero, M.; Adamowicz, L.; Chem. Phys. Lett. 2004, 387, 136.

40. Webb, S.; Out of This World: Colliding Universes, Branes, Strings and Other Wild Ideas of Modern Physics; Springer-Verlag: Berlin, 2004, p. 3 .

41. Laszlo, P.; Sci. Educ. 2013, 22, 1669.

42. Zuckerman, J. J.; J. Chem. Educ. 1986, 63, 829.

43. Shusterman, A. J.; Shusterman, G. P.; J. Chem. Educ. 1997, 74, 771.

44. Ogilvie, J. F.; J. Chem. Educ. 1990, 67, 280.

45. Lewars, E. G.; Computational Chemistry: Introduction to the Theory and Applications of Molecular and Quantum Mechanics, Springer International Publishing: Switzerland, 2016, pp. 121. 